\title{
FAST AND ADAPTIVE SURFACE RECONSTRUCTION FROM MOBILE LASER SCANNING DATA OF URBAN AREAS
}

\author{
M. Gordon, M. Hebel, M. Arens \\ Fraunhofer Institute of Optronics, System Technologies and Image Exploitation IOSB \\ Ettlingen, Germany \\ marvin.gordon@iosb.fraunhofer.de, marcus.hebel@iosb.fraunhofer.de, michael.arens@iosb.fraunhofer.de \\ http://www.iosb.fraunhofer.de
}

KEY WORDS: LIDAR, Mapping, Mobile laser scanning, Urban, Surface reconstruction

\begin{abstract}
:
The availability of 3D environment models enables many applications such as visualization, planning or simulation. With the use of current mobile laser scanners it is possible to map large areas in relatively short time. One of the emerging problems is to handle the resulting huge amount of data. We present a fast and adaptive approach to represent connected 3D points by surface patches while keeping fine structures untouched. Our approach results in a reasonable reduction of the data and, on the other hand, it preserves details of the captured scene. At all times during data acquisition and processing, the 3D points are organized in an octree with adaptive cell size for fast handling of the data. Cells of the octree are filled with points and split into subcells, if the points do not lie on one plane or are not evenly distributed on the plane. In order to generate a polygon model, each octree cell and its corresponding plane are intersected. As a main result, our approach allows the online generation of an expandable 3D model of controllable granularity. Experiments have been carried out using a sensor vehicle with two laser scanners at an urban test site. The results of the experiments show that the demanded compromise between data reduction and preservation of details can be reached.
\end{abstract}

\section{INTRODUCTION}

3D environment models are required for multiple purposes in the area of planning and simulation. One of the established ways to generate dense $3 \mathrm{D}$ models is the usage of laser scanners for the direct acquisition of 3D point clouds. In this paper we are especially interested in 3D models of urban environments. Vehicleborne mobile laser scanning (MLS) and direct georeferencing are well suited for fast and fine-grained acquisition of 3D data of urban areas, which can be performed independent of lighting conditions (e.g., even at night). The availability of such data is especially useful for the support of short-term operations. Examples can be found in the on-site acquisition, transmission and visualization of up-to-date 3D information to support rescue missions, emergency services, or disaster management.

In the last decade, mobile 3D laser scanners became available which provide billions of points in relatively short time. However, due to this large amount of data, current state of the art computer workstations are not even capable to visualize the resulting point clouds, not to mention the impossibility to transmit the raw data via a radio link. But at the same time, many of the measured data points are redundant, since they can be ascribed to common surfaces. Our approach aims at representing groups of points by planes, since planes are considered to be the typical geometric primitive occuring in urban areas. In combination with the plane-based representation, an octree data structure is used to handle the huge amount of data.

\section{RELATED WORK}

The surface reconstruction from point clouds is a long standing problem with different solutions. An overview is given e.g. in (Remondino, 2003). A related problem is the (iso)surface extraction from volumetric data (for example generated by computer

\footnotetext{
${ }^{*}$ Corresponding author.
}

tomographs). One class of surface reconstruction algorithms generates a mesh to connect the points of the point cloud (e.g. (Marton et al., 2009)). Another way is to generate an implicit function and to use a surface extraction method afterwards, like marching cubes (Lorensen and Cline, 1987). A similar approach is described in (Hoppe et al., 1992).

A different solution is the grouping of points, which have something in common, like forming a geometric primitive (e.g. plane or sphere), cf. (Vosselman et al., 2004). A region growing approach for plane extraction and a subsequent convex hull determination for polygon generation is presented in (Vaskevicius et al., 2007). To speed up the plane extraction, the methods in the following papers separate the points into cells, either grid or octree, and try to extract a plane per cell: A grid approach with RANSAC plane extraction is shown in (Hansen et al., 2006). Two similar solutions using an octree data structure are presented in (Wang and Tseng, 2004) and (Jo et al., 2013). Both try to extract a plane in one cell and divide the cell, until a sufficient solution is found. In (Wang and Tseng, 2004) least square plane fitting is used, followed by a merge-strategy during postprocessing. (Jo et al., 2013) exploit the regular pattern of the TOF camera for "microplane" estimation and check if all "microplanes" in a cell match, given an error bound.

\subsection{Contribution}

The approach presented in this paper combines and extends the ideas of methods listed in the previous section: It uses an octree as data structure and it combines the octree with a robust RANSAC plane extraction. This results in multiple benefits: The data structure can be dynamically increased and it adapts to local details. There is no need to assume a specific scan pattern since the approach is designed to handle each measured point independently. For this reason, new data can be entered into an existing model during the data acquisition. Additionally, an important feature of the approach is its adjustable high compression rate with moder- 
ate loss of information. This adjustability is achieved by setting an error bound for the local point-to-plane distance.

\section{METHOD}

At first an overview of our approach is given. Then the different parts are described in more detail.

\subsection{Overview}

The input to our method consists of directly georeferenced point clouds (single scans), where each cloud covers a part of the scene. Neither the size of the scene nor the number of point clouds need to be known. This is achieved by using a dynamically growing data structure and a method which handles every cloud in the same way. The main result is a polygon model, which represents the input points lying on planar surfaces. Also points not represented by the polygon model are given. This is the case if they cannot be represented by a plane (e.g. vegetation) or their neighborhood is too sparse.

The main data structure is an octree, which allows to be increased dynamically if needed. Its cell size has a lower bound, e.g., defined in accordance with the accuracy of the laser scanner or the positioning errors. It also permits a computationally cheap plane extraction. For every cell of the octree, the goal of our approach is to have either none or exactly one plane as a representative. The plane fitting is carried out by using a RANSAC (cf. (Fischler and Bolles, 1981)) approach including a refinement based solely on the set of inliers. If mainly outliers are found, that cell is further divided in eight subcells until a certain level of detail is reached (the minimal octree cell size). In case a representing plane can be determined, the data points should additionally be uniformly distributed among this plane. In our approach this test is done after a cell is kept untouched for a certain time.

The polygon model itself is created by computing the intersection of each cube (octree cell) with the plane assigned to it. This step can be done at any time.

In the next subsection we describe the details of the point handling. Afterwards the test of uniform distribution is explained. The section ends with a list of the involved parameters and a description of some implementation details.

\subsection{Point handling}

Our approach handles every point on its own, so there is no need to have the points organized in larger data chunks. However, in our experiments we always used point clouds representing single scans (rotations of the scanner head).

Assuming that a plane was already fitted to an octree cell, then all points in this cell are divided in a RANSAC manner into support (S) and contradiction set (C). If a new point is added to this cell, its destination set is determined based on the point-to-plane distance. In case of too many outliers, a new plane fit is carried out or finally, if there are still too many outliers, the octree cell is divided.

In case of a previously empty cell or a cell without plane (w.p.), the new point is added to a single set until enough points are collected in this cell. Then a plane fit is performed, and if it succeeded, the two sets (support and contradiction) are determined. Otherwise more points are collected in this cell and the plane fitting is retried. However, if a certain number of points is reached without successful plane fitting, the octree cell is divided.

The details are given as pseudo-code in Algorithm 1.

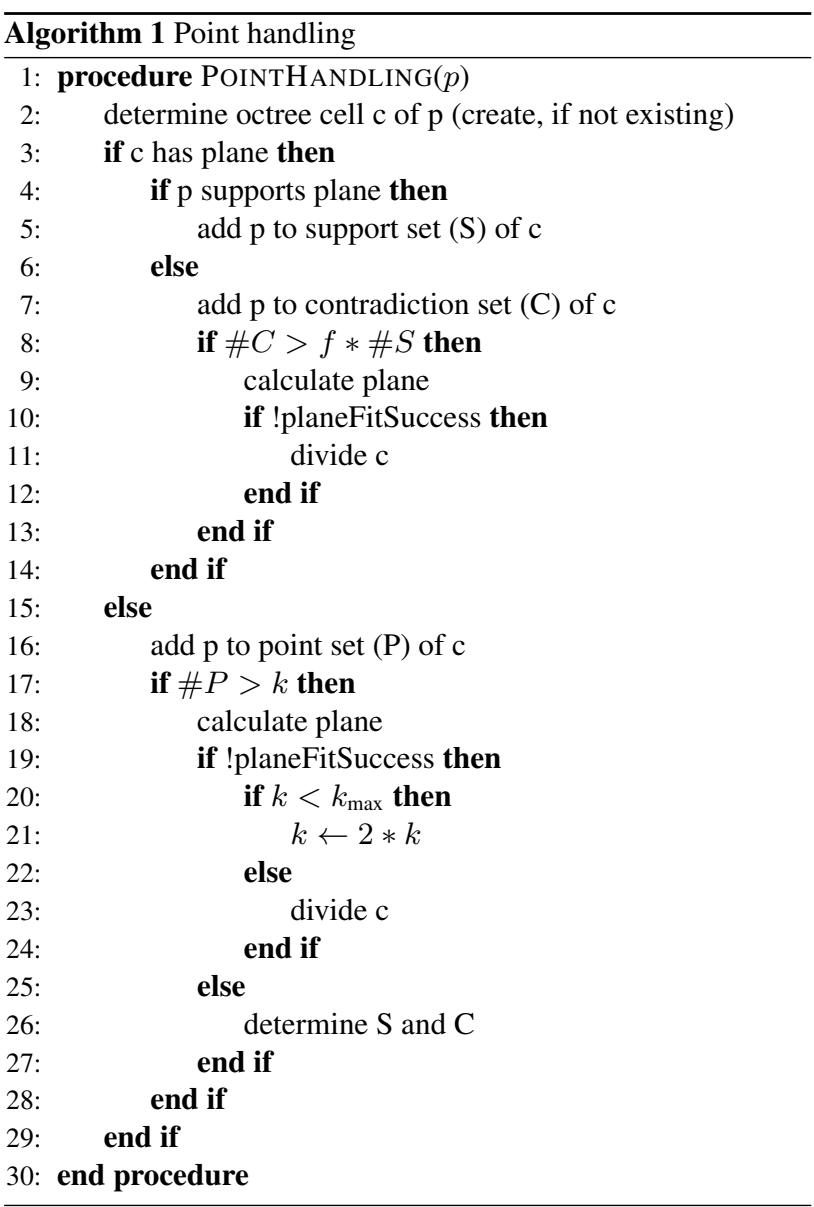

\subsection{Test for point-plane coverage}

This section describes the method that we use to test if the points in a given octree cell are uniformly distributed among the plane assigned to this cell. The reason to perform this test is to guarantee that the resulting planar patch will be a best possible representative of the points it is supposed to replace afterwards. If this constraint is violated, then the cell is further divided into eight subcells. To avoid too early cell subdivisions, each cell is tested only after a reasonable amount of time, in which it was no longer modified, e.g., if the cell received no more points for several rotations of the scanner head. As a "side-effect", a better estimation of the plane parameters is obtained.

The analysis of the point distribution is carried out using the principal component analysis (PCA) (e.g. (Hoppe et al., 1992)). It is only applied to the support set. Assuming that these points lie on a plane, then the two larger eigenvalues $\left(\lambda_{1}\right.$ and $\left.\lambda_{2}\right)$ represent the variance of the point distribution along the two major axes of the plane. The square root of the eigenvalues yields the corresponding standard deviation.

In case of a one-dimensional uniform distribution, the standarddeviation in the interval $[a, b]$ is $(b-a) /(2 \sqrt{3})$. Applied to our problem and under the assumption of nearly uniformly distributed points, $l /(2 \sqrt{3}) \approx 0.28 \cdot l$ (where $l$ stands for the cell side length) is an upper bound for the square root of the two bigger eigenvalues. To allow some variation, a smaller threshold is used as a test criterion $\left(\sqrt{\lambda_{1}}>t\right.$ and $\left.\sqrt{\lambda_{2}}>t\right)$. This threshold $t$ is called minimal variation.

\subsection{Parameters}

The approach presented here incorporates eight parameters. The following list explains these parameters: 
allowed distance Maximal allowed point-to-plane distance for both the point handling and the RANSAC plane fitting.

proportion of outliers Maximal allowed proportion of outliers for both the point handling (cf. $f$ in Algorithm 1) and the RANSAC plane fitting.

RANSAC iterations Number of RANSAC iterations during the plane fitting.

minimal cell size The minimal octree cell size.

$\boldsymbol{k}_{\text {start }}$ Minimal number of points needed in a cell before starting plane fitting (cf. $k$ in Algorithm 1).

$\boldsymbol{k}_{\max }$ Upper bound for $k$ (cf. $k_{\max }$ in Algorithm 1).

minimal variation This value $t$ is the criterion for uniform distribution of points (see section 3.3 for details)

test delay The number of scans (full rotations of the scanner head) with no modification of the specific octree cell. After that time the plane assigned to that cell is tested for uniform distribution of points.

\subsection{Details of the implementation}

All parts of our implementation take advantage of the freely available Point Cloud Library (PCL, http://pointclouds.org/) (Rusu and Cousins, 2011). Especially its octree implementation was used and extended where needed. The PCL itself uses the Visualization Toolkit (VTK, http://vtk.org/) for visualization purposes. We additionally utilize the VTK to calculate the intersection between an octree cell and a plane in order to get the resulting polygon.

\section{EXPERIMENTS}

\subsection{Experimental setup}

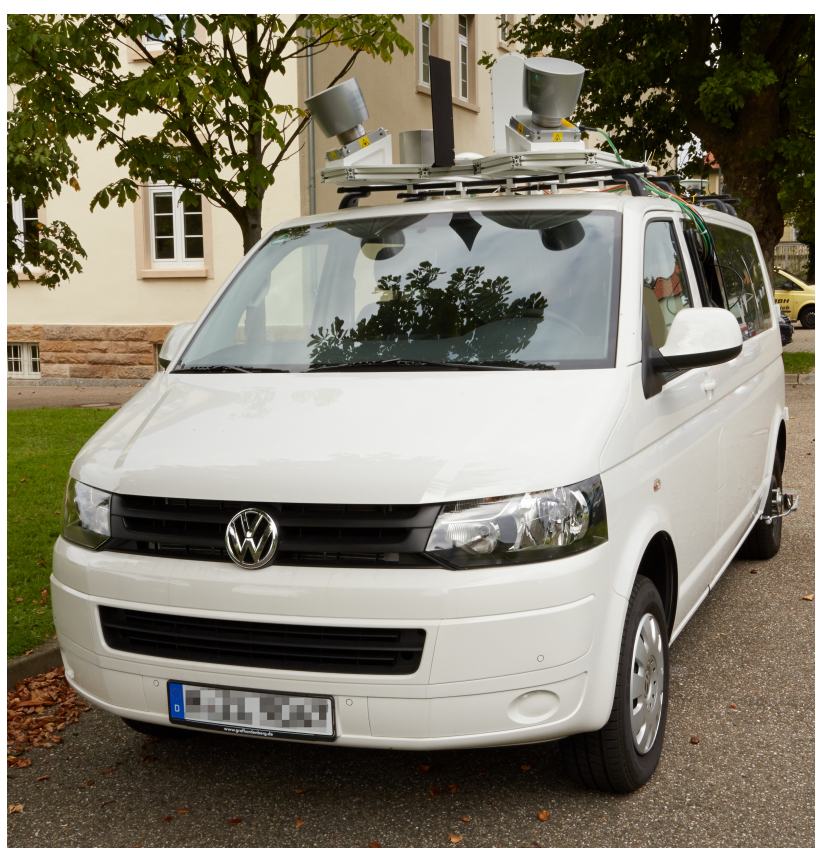

Figure 1. Sensor vehicle used for the experiments.

The data used for the experiments were recorded with a GNSS/INS augmented sensor vehicle (cf. Figure 1). On this vehicle, two Velodyne HDL-64E laser scanners are located over the front corners of the vehicle roof, and are positioned on a wedge with a 25 degree angle to the horizontal. This configuration guarantees a good coverage of the roadway in front of the car and allows scanning of building facades alongside and behind it. For direct georeferencing an Applanix POS LV inertial navigation system is built into the van. It comprises the following sensor components: an IMU (inertial measurement unit), two GNSS antennas and a DMI (distance measuring indicator).

Each one of the laser scanners has a rotation rate of $10 \mathrm{~Hz}$ and a data rate of approximately 1.3 million points per second. The navigation system has a data rate of $200 \mathrm{~Hz}$.

\subsection{Data acquisition}

GNSS (e.g., GPS) signals are - among other things - influenced by atmospheric and multipath effects. Within our experiments, we used additional GNSS data of nearby reference stations to reduce the impact of atmospheric effects. This can be done in realtime (RTK) as well as during postprocessing, and it results in a more accurate and precise position estimation. In this paper, the inclusion of precise GNSS reference data allowed us to focus on the model generation without having to deal with localization and data alignment issues. However, in future work, the presented methods for model generation will be supplemented by simultaneous localization and mapping (SLAM) techniques.

Prior to the actual measurements, the two laser scanners have been calibrated by a procedure presented in (Gordon and Meidow, 2013) to achieve best possible precise point measurements. The transformation between the coordinate systems of the two scanners was determined by using the ICP algorithm (Besl and McKay, 1992). The relative orientation and position of the laser scanners with respect to the navigation system was calculated by using the approach presented in (Levinson and Thrun, 2010). For each single laser range measurement, the position and orientation of the vehicle was calculated by linear interpolation from the $200 \mathrm{~Hz}$ navigational data.

\subsection{Test site}

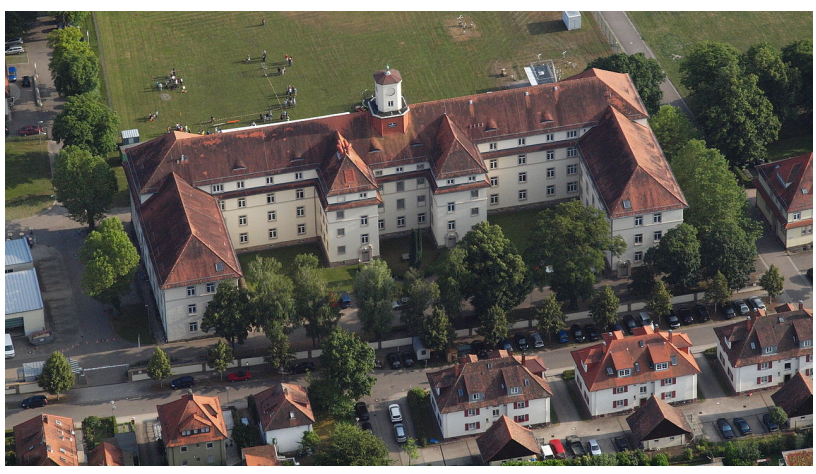

Figure 2. Aerial view of the test site ${ }^{1}$

The data acquisition took place at a small test site, which is the outside area around our institute building, partly shown in Figure 2. The main part of the scene is a big building surrounded by neighboring structures and some trees. The path driven during the measurements is shown in Figure 3. The data acquisition took about 2.5 minutes and resulted in approximately 250 million $3 \mathrm{D}$ points. This value is smaller than the actual data rate of

\footnotetext{
${ }^{1}$ Ettlingen, Fraunhofer Institut IOSB by Wolkenkratzer http:// commons.wikimedia.org/wiki/File:Ettlingen,_Fraunhofer_ Institut_IOSB.JPG under http://creativecommons.org/ licenses/by-sa/3.0
} 
the laser scanners, since some points measured in close vicinity of the sensors (e.g., the vehicle roof) were filtered out during the point generation process.

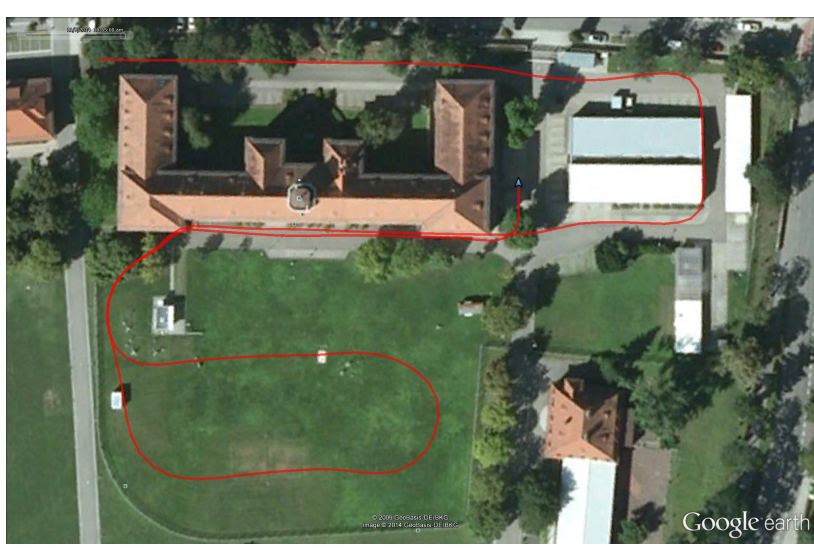

Figure 3. Path driven by the sensor vehicle during the data acquisition (Image data: Google Earth, Image (c) 2014 GeoBasis$\mathrm{DE} / \mathrm{BKG})$.

A part of the measured points is shown in Figure 4.

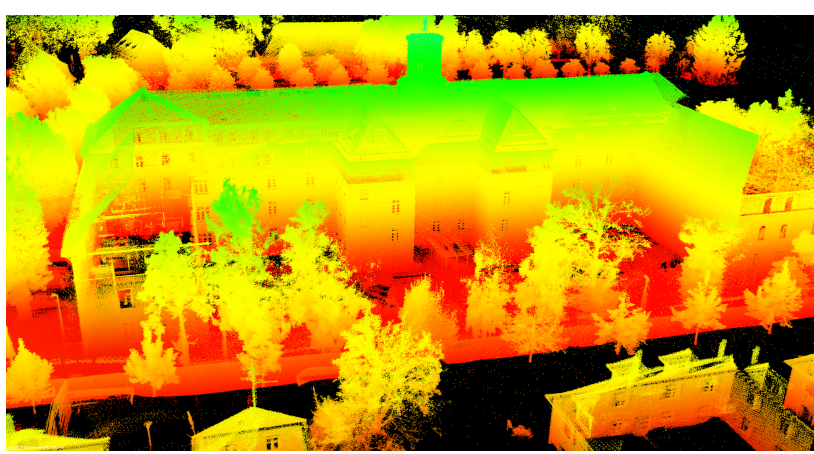

Figure 4. Plotted accumulated 3D data after reduction to a voxel grid (for visualization).

\section{RESULTS AND DISCUSSION}

The previous section described the experimental setup and the acquisition of data used for the experiments. We carried out different runs of the algorithms with these data to investigate, analyze and validate our approach. In each run one of three parameters was altered. For the other parameters a default value was set. Table 1 lists the default values for all parameters. The evaluated three parameters are the allowed distance, the proportion of outliers and the minimal variation. The reconstruction error is not considered for the evaluation, because it is directly linked to the allowed distance-parameter, which is an upper bound for the reconstruction error.

\begin{tabular}{|c|c|}
\hline parameter & value \\
\hline allowed distance & $0.25 \mathrm{~m}$ \\
proportion of outliers & 0.2 \\
RANSAC iterations & 100 \\
minimal cell size & $0.1 \mathrm{~m}$ \\
$k_{\text {start }}$ & 50 \\
$k_{\max }$ & 201 \\
minimal variation & 0.2 \\
test delay & 10 scans \\
\hline
\end{tabular}

Table 1. Default values for the parameters.

Figure 5(a) shows a part of the polygon model (without the remaining single points), which resulted from applying our method with the default parameter settings to the acquired data set. It is visualized from the same viewpoint as Figure 4. Most of the polygons have a quadratic shape. After applying our approach, the surface of the model is not continuous, because there typically is a small gap between neighboring polygons. Especially the trees lead to many small and unconnected polygons, which have a nearly random orientation.

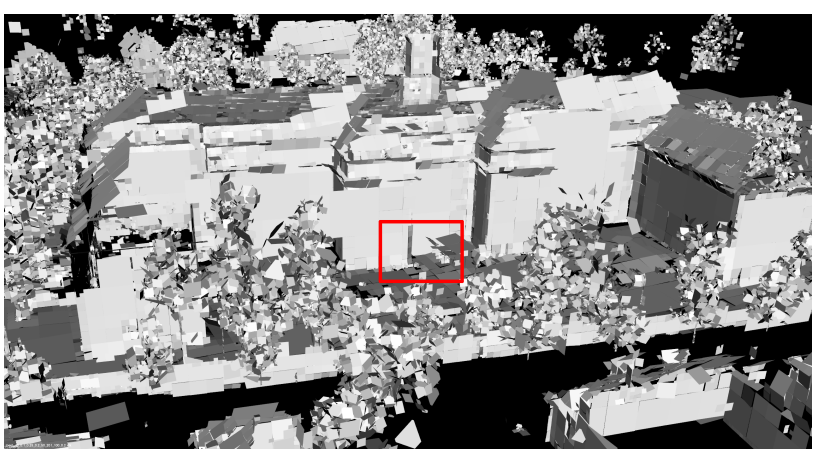

(a) Overview

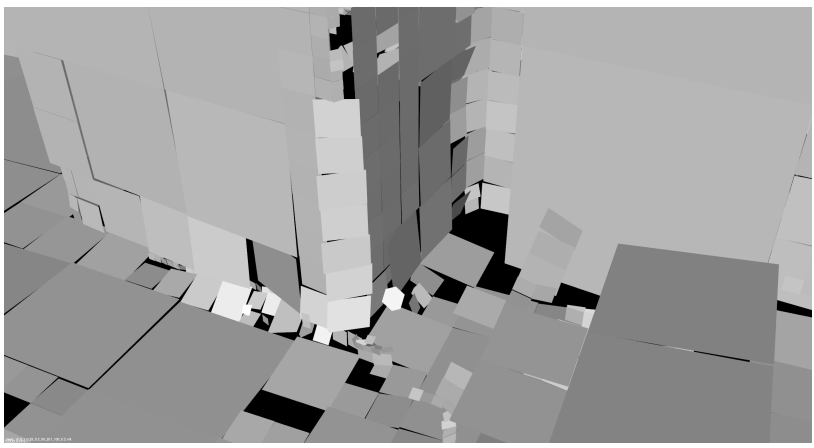

(b) Zoomed area

Figure 5. Polygon model generated with the default parameter settings. The content of the area marked in Figure (a) is shown zoomed in by Figure (b).

\subsection{Variation of the allowed distance}

In the first experiment the allowed point-to-plane distance was altered in the range from $1 \mathrm{~cm}$ to $100 \mathrm{~m}$. The resulting space savings are shown in Figure 6. Here the term space savings means the proportion of saved data to the original amount of data and is calculated with equation (1). Throughout this paper, the terms space savings and compression rate are used synonymously.

$$
s=1-\frac{\# \text { points mesh }+\# C+\# \text { points in cells w. p. }}{\# \text { points }}
$$

In Figure 6 the abscissa is plotted with a logarithmic scale. The compression rate obviously increases up to $90 \%$ until an allowed distance of $0.1 \mathrm{~m}$. For higher values the compression rate keeps nearly stable, between an allowed distance of $0.5 \mathrm{~m}$ and $100 \mathrm{~m}$ there is only little change.

The point handling (cf. Algorithm 1) and the test for uniform distribution (cf. section 3.3) are the most time consuming parts. Therefore the calculation of the intersection between octree cells and their associated planes is excluded from the runtime assessment. Figure 7 depicts the measured runtimes. The graph shows a nearly opposite behavior when compared to that of the data compression rate: First the runtime clearly decreases until an allowed distance of $0.25 \mathrm{~m}$, followed by a parameter range with nearly 


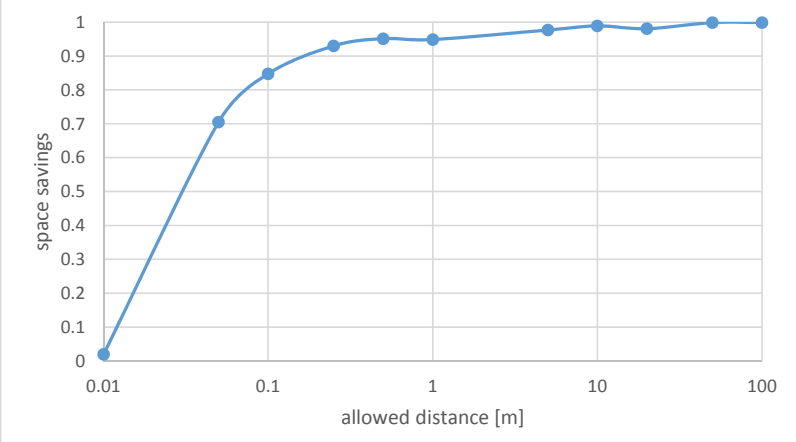

Figure 6. Resulting space savings for different allowed point-toplane distances.

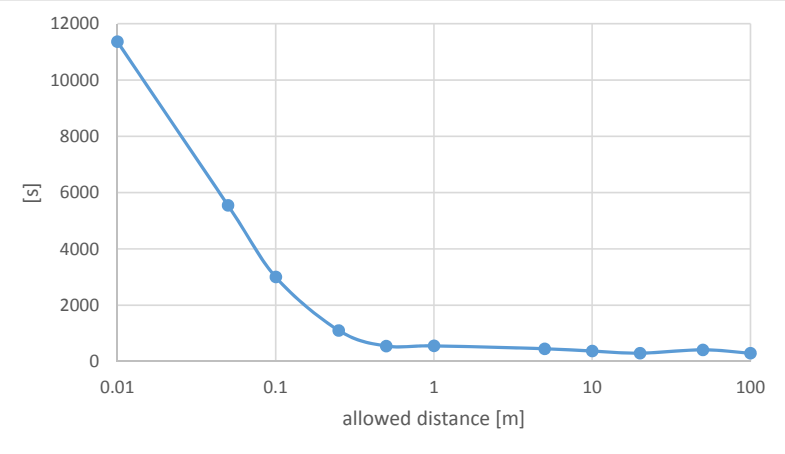

Figure 7. Runtime for different allowed distances.

stable runtime values. The plotted time for an allowed distance of $0.01 \mathrm{~m}$ equals to approximately 3 hours, followed by $18 \mathrm{~min}$ utes for $0.25 \mathrm{~m}$ and 5 minutes for $100 \mathrm{~m}$. However, these values are specific to our (still not optimal) implementation, the methods are appropriate to run in realtime on an operational system.

The results show a close relation between computation time and data compression rate. The reason for the high computation time in case of small values for the allowed distance is the frequent need for plane fitting and octree cell division. With a higher bound, the points can simply be added to existing planes. This reduces the number of octree cells without plane and therefore results in better compression rates. However, this also leads to a higher loss of details. In an extreme case (e.g., allowed distance of $100 \mathrm{~m}$ ) nearly all points of the data set are represented by only one plane, which of course is not the desired result.

If the scene contains a lot of planes, the precision and accuracy of the point positioning have a significant influence on the compression rate, given a certain allowed distance. If the allowed distance is too small, most measured $3 \mathrm{D}$ points remain untouched and the compression rate is poor. In our case, the precision and accuracy of the point positioning process are in a combined magnitude of approximately $20 \mathrm{~cm}$.

For the following experiments a tradeoff between space savings, runtime behavior and level of detail is needed. We decided to set the allowed distance to $0.25 \mathrm{~m}$ for all other experiments.

\subsection{Variation of the proportion of outliers}

For the second experiment, the maximum allowed proportion of outliers was varied between $1 \%$ and $90 \%$.

The resulting proportion of the different point sets is shown in Figure 8 . If a cell contains a plane, the number of points in each

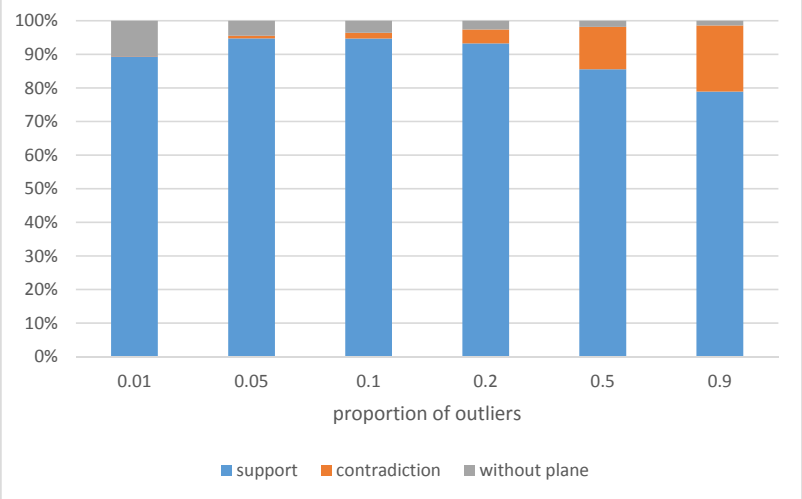

Figure 8. Proportion of the different point sets.

set (support set, contradiction set) is counted. In case of an octree cell without plane, its number of points increases the "without plane" counter. The average proportion of support sets is an indicator for the possible space savings, since the associated points are supposed to be represented by planes.

In the case of only one percent of allowed outliers, there are virtually no outliers and over 10 percent of the points lie in cells without plane. On the other hand, with 90 percent allowed outliers, there are (on an average) about 20 percent outliers and only very little points in cells without plane. With the data used in our experiments, the optimum value of the average support set size was reached with a proportion of outliers of 5 percent.

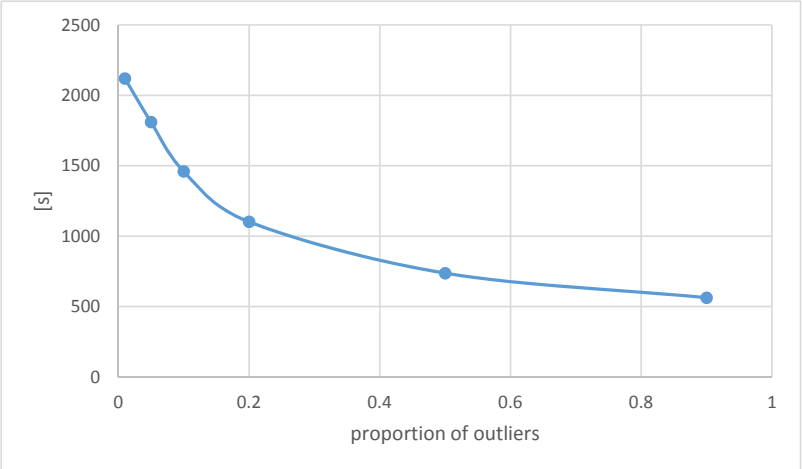

Figure 9. Runtime for different proportions of outliers.

Obviously there is a negative correlation between runtime and the setting of the proportion of outliers (cf. Figure 9). The curve has a higher slope between $1 \%$ and $20 \%$ proportion of outliers and a smaller slope between $20 \%$ and $90 \%$. The runtime in our experiments varied between 35 minutes and 10 minutes.

The compression rate decreases with higher outlier rate, since the outlying points represent $3 \mathrm{D}$ details in the scene and only the support set points can be replaced by planes. If the space savings and the runtimes are compared, the proportion of outliers has a higher influence on the runtime. It decreases from 35 minutes to 9 minutes, which is a reduction of $75 \%$. The compression rate only shows a reduction of $15 \%$.

\subsection{Changing parameter setting of minimal variation}

To show the impact of the test for point-plane coverage, the model was created with the minimal variation setting varied between 0.1 and 0.25 . Figure 10 (a) and 10 (b) show screenshots of parts of the resulting polygon model. One clearly visible difference between these figures is: in Figure 10(a) there is a big polygon marked 
in red with points only on $70 \%$ of its right side. In Figure 10(b) this big polygon is missing and its area is instead represented by smaller ones, which are nearly totally filled with points. In addition, there are areas where no polygon was assigned to the points. These areas are shown as black parts in Figure 10(b). An exception are the window areas of the building facades, that are covered by polygons. Presumably these areas are too small and only have a minor impact on the standard deviations (cf. subsection 3.3). In summary, with a higher value for the minimal variation the point variation test reduces the cell size down to its minimal allowed setting, so that the points are nearly spread over the whole plane or polygon, respectively.

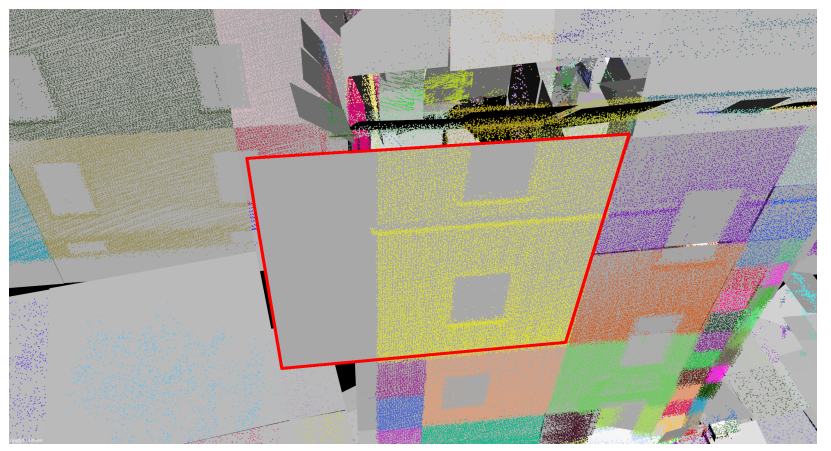

(a) 0.1

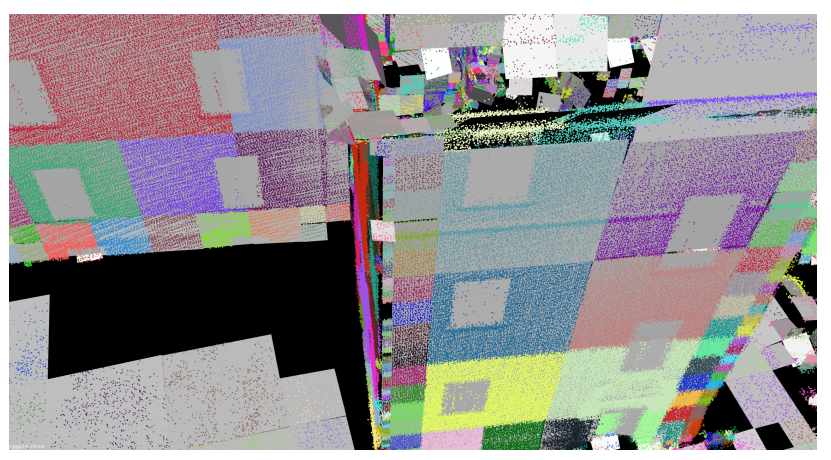

(b) 0.25

Figure 10. Parts of the polygon models and the points in the support sets (thinned for visualization) for different minimal variations (points of the same cell have the same color).

\section{CONCLUSIONS AND FUTURE WORK}

We have presented an approach for adaptive and fast surface reconstruction based on MLS data acquired in urban areas. It takes points as input and tries to represent them by planes, which results in a lossy but efficient data compression. Especially the influence of the two parameters allowed distance and proportion of outliers was analyzed. Both parameters have an important influence on the computation time. The data compression rate is influenced by both, but the allowed distance showed a more important influence. In combination with the minimal allowed cell size, these parameters control the level of detail of the resulting (polygon) model. This allows the usage for the intended application: The polygon model can be rendered in real-time and therefore allows the inspection e.g. by emergency services. The compression rate of more than $90 \%$ enables an easier and faster transmission.

Future work will focus on different areas: Vegetation in the scene results in many small and randomly orientated polygons (cf. section 4.). A better representation could consist of thinned points which are marked as vegetation. In some cases the possibility of cell fusion could gain better space savings. The implementation of the approach needs to be optimized. In this context, we plan to implement parts of the algorithms (e.g., the test of uniform distribution) to run on multiple CPUs. Texturing the model with RGB data will be an additional extension.

We will supplement the 3D model generation with realtime 3DSLAM techniques, which will especially be useful for indoor applications where no GNSS-signals are available. Even in the outdoor case, we observed inaccurate GNSS data due to multipath effects. So we expect to clearly benefit from SLAM techniques even in that case, which will result in a better model accuracy and precision.

\section{References}

Besl, P. and McKay, N. D., 1992. A method for registration of 3-D shapes. IEEE Transactions on Pattern Analysis and Machine Intelligence 14(2), pp. 239-256.

Fischler, M. A. and Bolles, R. C., 1981. Random sample consensus: a paradigm for model fitting with applications to image analysis and automated cartography. Communications of the ACM 24, pp. 381395 .

Gordon, M. and Meidow, J., 2013. Calibration of a multi-beam Laser System by using a TLS-generated Reference. ISPRS Annals of Photogrammetry, Remote Sensing and Spatial Information Sciences II5/W2, pp. 85-90.

Hansen, W. v., Michaelsen, E. and Thönnessen, U., 2006. Cluster Analysis and Priority Sorting in Huge Point Clouds for Building Reconstruction. In: 18th International Conference on Pattern Recognition (ICPR'06), pp. 23-26.

Hoppe, H., DeRose, T., Duchamp, T., McDonald, J. and Stuetzle, W., 1992. Surface Reconstruction from Unorganized Points. In: Proceedings of the 19th Annual Conference on Computer Graphics and Interactive Techniques, SIGGRAPH '92, ACM, pp. 71-78.

Jo, Y., Jang, H., Kim, Y.-H., Cho, J.-K., Moradi, H. and Han, J., 2013. Memory-efficient real-time map building using octree of planes and points. Advanced Robotics 27(4), pp. 301-308

Levinson, J. and Thrun, S., 2010. Unsupervised calibration for multibeam lasers. In: International Symposium on Experimental Robotics.

Lorensen, W. E. and Cline, H. E., 1987. Marching Cubes: A High Resolution 3D Surface Construction Algorithm. In: Proceedings of the 14th Annual Conference on Computer Graphics and Interactive Techniques, SIGGRAPH '87, ACM, pp. 163-169.

Marton, Z. C., Rusu, R. B. and Beetz, M., 2009. On Fast Surface Reconstruction Methods for Large and Noisy Datasets. In: IEEE International Conference on Robotics and Automation (ICRA), pp. 32183223 .

Remondino, F., 2003. From point cloud to surface: the modeling and visualization problem. International Archives of Photogrammetry, Remote Sensing and Spatial Information Sciences 34(5), pp. W10.

Rusu, R. B. and Cousins, S., 2011. 3D is here: Point Cloud Library (PCL). In: IEEE International Conference on Robotics and Automation (ICRA), Shanghai, China, pp. 1-4.

Vaskevicius, N., Birk, A., Pathak, K. and Poppinga, J., 2007. Fast detection of polygons in $3 \mathrm{~d}$ point clouds from noise-prone range sensors. In: Safety, Security and Rescue Robotics, 2007. SSRR 2007. IEEE International Workshop on, pp. 1-6.

Vosselman, G., Gorte, B. G. H., Sithole, G. and Rabbani, T., 2004. Recognising structure in laser scanner point clouds. International Archives of Photogrammetry, Remote Sensing and Spatial Information Sciences 46(8), pp. 33-38.

Wang, M. and Tseng, Y.-H., 2004. Lidar data segmentation and classification based on octree structure. International Archives of Photogrammetry, Remote Sensing and Spatial Information Sciences 20(B3), pp. 6. 